\title{
STEM of a Single Crystal Lithium Ion Battery Anode during Electrochemical Cycling
}

Jared J. Lodico $^{1 *}$, Matthew Mecklenburg ${ }^{2}$, Ho Leung Chan ${ }^{1}$, William A. Hubbard ${ }^{1}$, and B. C. Regan ${ }^{1}$

1. Department of Physics \& Astronomy and California NanoSystems Institute, University of California, Los Angeles, USA

2. Core Center of Excellence in Nano Imaging, University of Southern California, Los Angeles, USA

* Corresponding author: jaredlodico@ucla.edu

The lithium ion battery (LIB) is currently the preferred power source for most portable applications. Despite the LIB's importance, in situ observation of the cycling process at small length scales still remains an open problem in the research community. As a LIB is charging, lithium ions are electrochemically inserted, or intercalated, between the graphene layers of the graphite anode, creating a lithium-carbide structure [1]. Little is known, even in the ideal case, about this process at the macromolecular level. Liquid cell based in situ transmission electron microscopy is a promising route to reveal lithium kinetics, electrode/electrolyte interactions, and rate limiting physics [2, 3]. Here, we use in situ scanning transmission electron microscopy (STEM) to image the electrochemical intercalation of lithium in single crystal graphite and observe a key failure mode in LIBs.

The in situ fluid cells consist of two $\mathrm{Si} / \mathrm{SiO}_{2}$ chips, each with an electron-transparent, $\mathrm{Si}_{3} \mathrm{~N}_{4}$ window, sandwiched together back-to-back. Natural graphite (NGS Naturagraphit GmBH) is mechanically exfoliated and wet-transferred to an electron-transparent window in a silicon chip. The window is equipped with a Ti/Pt $(5 / 25 \mathrm{~nm})$ electrode patterned via optical lithography. A thin conformal capping layer $(10-20 \mathrm{~nm})$ of $\mathrm{Al}_{2} \mathrm{O}_{3}$ deposited via atomic layer deposition encapsulates most of the exposed $\mathrm{Pt}$ electrode. This capping layer reduces unwanted chemistry on the Pt and minimizes its influence on the electronic transport data. A small droplet of electrolyte $\left(1 \mathrm{M}\right.$ lithium perchlorate, $\mathrm{LiClO}_{4}$, in ethyl carbonate and dimethyl carbonate, EC:DMC, mixed at a 1 to 1 volume ratio) is placed on the chip with the electrode and the graphite. (We find this electrolyte to be more beam-stable than alternatives studied by other authors [4].) The second, smaller chip is then placed on top of the droplet and manipulated to align the two windows, sandwiching the graphite and a thin layer of electrolyte between them. Finally, the two chips are sealed together with epoxy, creating a vacuum-compatible fluid cell.

A Gamry Reference 600 potentiostat is used to electrochemically intercalate a graphite flake via cyclic voltammetry $(\mathrm{CV})$. The cell potential is cycled at $10 \mathrm{mV} / \mathrm{s}$ from its open circuit potential (OCP), $-0.9 \mathrm{~V}$, to $-4 \mathrm{~V}$ (Fig. 1a). Both the pseudo-reference and counter electrodes are platinum. We use a Hummingbird Scientific biasing holder to electrically contact the fluid cell, and we image the sample in an FEI Titan 80-300 S/TEM using an accelerating voltage of $300 \mathrm{kV}$. Graphite in the electrolyte chosen is an excellent system for STEM study, as it is nearly immune to degradation in the electron beam: imaging for hours with 50 pA beam currents shows little beam-induced sample damage.

Before any electrochemical bias, bright field (BF) STEM of the graphite flake shows it to be pristine, with uniform contrast (Fig. 1b). The Pt electrode is visible in the top left of the frame. As the flake reaches an intercalating potential (Fig. 1a), it changes contrast and shows a variety of bright and dark bands (Fig. 1c - the flake is outlined in yellow) that are associated with intercalation [3]. In the subsequent frame (Fig. 1d), before the graphite can fully intercalate, circular dendrite structures, $100-$ 
$200 \mathrm{~nm}$ in diameter, form on the graphite's surface. These dendrites grow rapidly and use lithium that would otherwise be available for intercalation. After the potential ramp is reversed, the dendrites continue to grow, reaching their maximum size (Fig. 1e) $200 \mathrm{mV}$ later. Even after the graphite is returned to the OCP, the dendrites still coat the flake (Fig. 1f). In an actual LIB such a coating would compete with the graphite for lithium ions, and thus would lead to decreased capacity, lifetime, and safety [5].

\section{References:}

[1] MS Dresselhaus and G Dresselhaus, Adv. Phys. 51 (2002), p. 1-186.

[2] JM Tarascon and M Armand, Nature 414 (2001), p. 359.

[3] ER White, JJ Lodico, and BC Regan, Nature Communications 8 (2017).

[4] P Abellan et al., Nano Letters 14 (2014), p. 1293-1299.

[5] This work was supported by the National Science Foundation (NSF) Science and Technology Center (STC) award DMR-1548924 (STROBE).
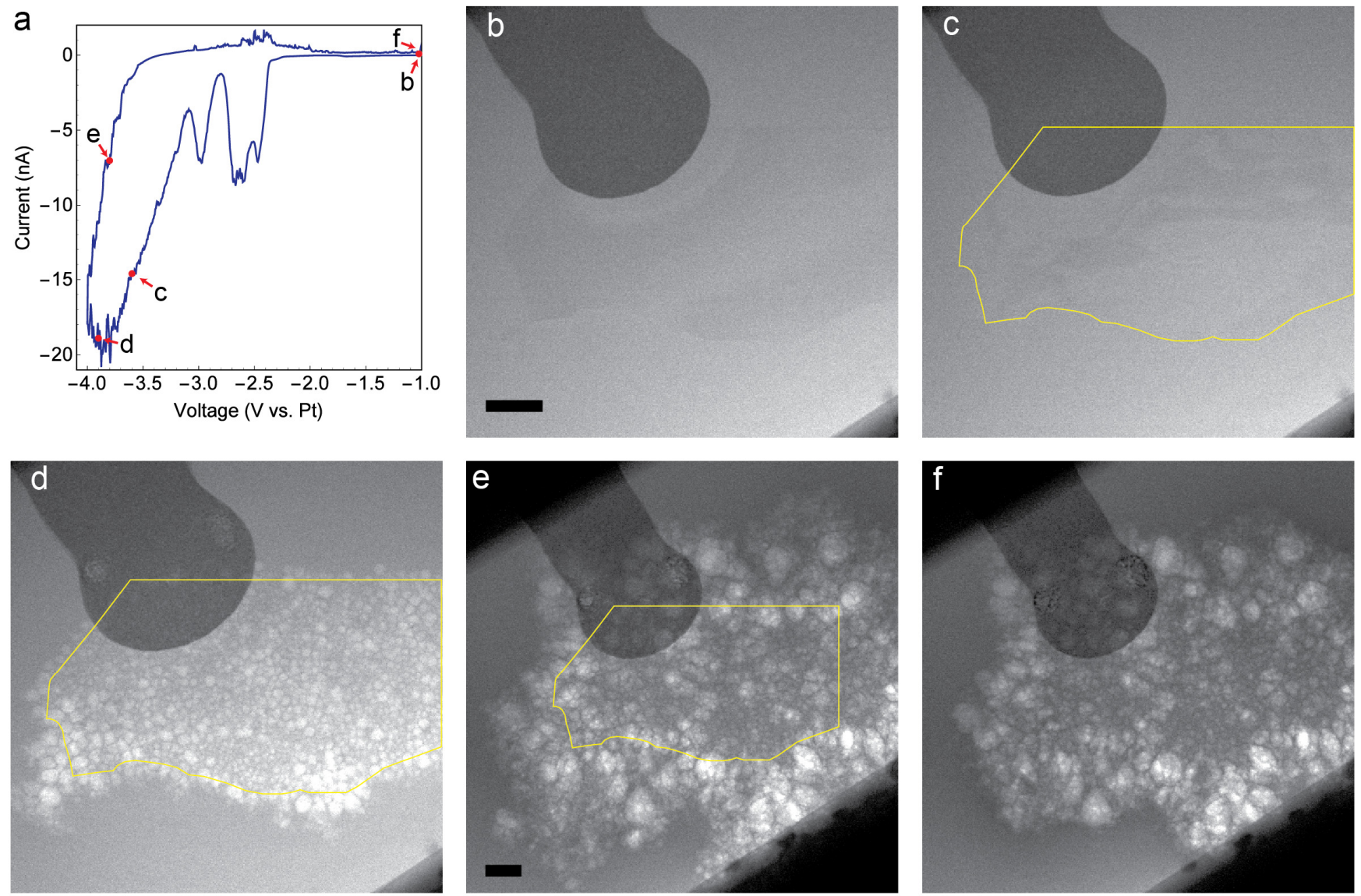

Figure 1. (a) Cyclic voltammogram acquired in situ. (b-f) Bright field STEM images of a graphite flake in a fluid cell during electrochemical cycling. The flake is electrically connected to a Ti/Pt $(5 / 25 \mathrm{~nm})$ electrode seen in the top left. The voltage and measured current for each frame (b-f) is highlighted in (a) by a red dot and arrow. A yellow line delineates the graphite flake's edge in (c-e). Each $512 \times 512$ pixel frame was acquired in $30 \mathrm{~s}$. The scale bars in (b) and (e) are $1 \mu \mathrm{m}$. 